\title{
Combination treatment of FTY720 and cisplatin exhibits enhanced antitumour effects on cisplatin-resistant non-small lung cancer cells
}

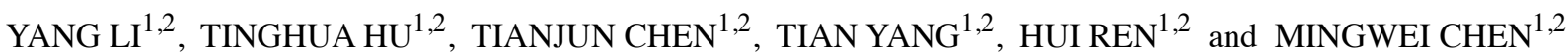 \\ ${ }^{1}$ Department of Respiratory and Critical Care Medicine, The First Affiliated Hospital of Xi'an Jiaotong University, \\ Xi'an, Shaanxi 710061; ${ }^{2}$ Shaanxi Provincial Research Center for the Project of Prevention and \\ Treatment of Respiratory Diseases, Xi'an, Shaanxi, P.R. China
}

Received March 25, 2017; Accepted October 9, 2017

DOI: 10.3892/or.2017.6111

\begin{abstract}
A major common medical treatment for lung carcinoma is cisplatin (DDP)-based therapy. However, the development or existence of chemoresistance frequently blocks its effectiveness. Currently, autophagy is recognised as a potential anticancer strategy, although there is controversy over its role in the development of cancer. In lung carcinoma, no studies of autophagy induced by FTY720, a sphingosine 1-phosphate analog and a novel immunosuppressant drug, have been published, while apoptosis has been shown to be induced by FTY720 in several cancer cell lines. We evaluated the effects of FTY720 on autophagy in A549 cells and studied the related mechanisms of cell autophagy and apoptosis in non-small cell lung carcinoma, including both DDP-resistant and -sensitive cells. The results revealed that FTY720 inhibited the growth and induced apoptosis in the A549/DDP cells in a time- and dose-dependent manner and the combination of FTY720 and DDP further enhanced apoptosis in these cells as determined by CCK-8 assay, western blotting and flow cytometry. Compared with the sensitive cell line A549, DDP-resistant A549/DDP cells showed a substantial increase in baseline autophagy as determined by increased LC puncta, and expression of LC3-I, LC3-II and Atg7 expression. DDP-induced apoptotic cell death was enhanced by the blockade of either siRNA-mediated knockdown of Atg7 genetic expression or a pharmacological inhibitor (3-MA). Moreover, the combination of FTY720 and DDP showed enhanced antitumour activity in vivo in lung cancer-bearing mice. Immunohistochemistry showed that the mice with lung carcinoma treated with FTY720 and DDP showed decreased expression of Atg7 and Ki67. Compared with monotherapy in vivo and in vitro, FTY720 in combination
\end{abstract}

Correspondence to: Professor Mingwei Chen, Department of Respiratory and Critical Care Medicine, The First Affiliated Hospital of Xi'an Jiaotong University, 277 West Yanta Road, Xi'an, Shaanxi 710061, P.R. China

E-mail: chenmw0320@163.com

Key words: FTY720, cisplatin, resistance, autophagy, non-small lung cancer cells with DDP inhibited A549 cell growth more effectively. and these findings also show the influence of FTY720 in the induction of autophagy. Overall, the results indicated that FTY720 in combination with a DDP-based regime could enhance the effectiveness of lung carcinoma treatment.

\section{Introduction}

In China and many other countries, lung carcinoma is a leading cause of cancer-related death (1). Nearly $70-80 \%$ of all lung carcinoma cases are classified as non-small cell lung cancer (NSCLC), including large and squamous cell carcinoma, as well as adenocarcinoma (2). Currently, chemotherapeutic agents are commonly used for the treatment of lung carcinoma. A platinum-based compound, cisplatin (DDP), is one of the leading chemotherapeutic agents for NSCLC treatment $(3,4)$. However, the development of chemoresistance frequently blocks its effectiveness $(5,6)$. Therefore, elucidation of the molecular mechanisms of DDP resistance may be helpful for clinicians to identify resistance earlier and, consequently, improve the effectiveness of lung carcinoma treatment. Thus, identifying novel agents with anticancer effects on DDP-resistant lung carcinoma cells is desperately needed.

FTY720 [2-amino-2-[2-(4-octylphenyl)-1,3-propanediol hydrochloride)], which is also known as Gilenya or fingolimod, is an artificial compound based on the secondary fungal metabolite myriocin (ISP-I) and is an effective immunosuppressant that was validated as a new treatment for multiple sclerosis by the FDA in September 2010. FTY720 is a potential treatment for organ transplantation and cancer and has been assessed in many types of cancer, including ovarian cancer (7), hepatocellular carcinoma (HCC) (8), multiple myeloma (9), leukaemia (10), glioblastoma (11) and prostate cancer (12). Studies conducted in pre-clinical models have also been performed. Current evidence suggests that FTY720 has effective anticancer action in carcinoma models, but the mechanisms of cancer cell death mediated by FTY720 have been reported to change based on cancer type.

Currently, autophagy is recognised as a potential anticancer strategy, although there is controversy over its role in the development of cancer (13). Autophagy was first recognised as an evolutionarily conserved process of catabolism by which cells 
control protein turnover and eliminate damaged organelles as well as misfolded proteins (14). It is an adaptive reaction to multiple types of stress, including oxidative and metabolic stress, which occurs not only in cancer cells, but also in normal cells (15). Maintaining energy homeostasis by recycling cellular components is the primary role of autophagy (16). The influence of this process on cancer chemosensitivity and tumourigenesis, including that of lung cancer, is unclear, although it promotes the survival of normal cells.

In lung carcinoma, no studies of autophagy induced by FTY720 have been published, while apoptosis has been shown to be induced by FTY720 in several cancer cell lines. We evaluated the effects of FTY720 on autophagy in A549 cells and studied the related mechanisms of cell autophagy and apoptosis, which are different cell death pathways.

\section{Materials and methods}

Cell culture and reagents. The American Type Culture Collection (ATCC; Manassas, VA, USA) provided the human lung carcinoma cell lines. Dulbecco's modified Eagle's medium (DMEM) containing $20 \mathrm{mM}$ HEPES buffer and $100 \mu \mathrm{g} / \mathrm{ml}$ gentamycin as well as $10 \%$ foetal bovine serum (FBS) was used to culture the cells for all experiments. FTY720 was obtained from Echelon Biosciences (Salt Lake City, UT, USA). Anti-Atg7, anti-GAPDH, anti-Bax, anti-Bcl-2 and anti-cleaved caspase-9 as well as antiLcs3 antibodies were purchased from Abcam (Cambridge, UK).

Cell Counting Kit-8 (CCK-8) assays. Cancer cells were seeded in $100 \mu 1$ of growth medium at a density of $8 \times 10^{3}$ cells/well in 96-well plates. Cells were treated with the indicated regents or left untreated after incubation overnight. Then, $10 \mu \mathrm{l} \mathrm{CCK-8}$ solution (CK04; Dojindo Laboratories, Kumamoto, Japan) was added to every well for $2 \mathrm{~h}$ every $24 \mathrm{~h}$. A microplate reader (BioTek Instruments, Inc., Winooski, VT, USA) was used to assess the viable cells, and absorbance was read at $595 \mathrm{~nm}$. Viable cells were analysed and compared with the non-drugtreated group. The results are expressed as a percentage of the control viable cells.

Flow cytometric analysis. In 60-mm plates, A549 cells were cultured and treated with specific reagents. Control small interfering RNA (siRNA) (siCON) or control reagents acted as negative controls. The cells were suspended again in buffer solution (100 mM NaCl, pH 7.4, $100 \mathrm{mM}$ HEPES and $25 \mathrm{mM}$ $\mathrm{CaCl}_{2}$ ) with $12.5 \mu \mathrm{g}$ of RNase, and incubated for another $30 \mathrm{~min}$ at $37^{\circ} \mathrm{C}$ for flow cytometric analysis. In the dark, $10 \mu \mathrm{l}$ of a propidium iodide (PI) solution $(50 \mu \mathrm{g} / \mathrm{ml})$ and $100 \mu \mathrm{l}$ of Annexin V-FITC were added to the cells for $30 \mathrm{~min}$ at room temperature, and then, the cells were stained. The resultant cells were labeled by Annexin V-FITC and PI and analyzed on fluorescence-activated cell-sorting (FACS) Calibur (BD Biosciences, San Jose, CA, USA) with FlowJo software version 10. All tests were carried out 3 times.

Cell transient transfection. Transfection was carried out with Lipofectamine 2000 Transfection Reagent in accordance with the manufacturer's procedure (Invitrogen, Carlsbad, CA, USA). The day before transfection, cells were seeded in 6 -well plates. A 100-pmol sample of siCON or siAtg7 in $250 \mu 1$
Opti-MEM medium (Gibco, Grand Island, NY, USA) was mixed with $5 \mu$ l Lipofectamine 2000 dissolved in $250 \mu \mathrm{l}$ of the same medium and allowed to stand at room temperature for $20 \mathrm{~min}$. The resulting $500 \mu \mathrm{l}$ transfection solutions were then added to each well, which already contained $1.5 \mathrm{ml}$ of Opti-MEM. After $6 \mathrm{~h}$, the cultures were replaced with $2 \mathrm{ml}$ fresh DMEM medium. Atg7 was targeted with siRNA duplexes (siAtg7) targeting the following sequences: 5'-CCAACACACU CGAGUCUUU-3' and 5'-GCCCACAGAUGGAGUAGCA-3'. A non-targeting siRNA (siCON) was used as a control with sense (5'-UCUACGAGGC ACGAGACUU-3') and antisense (5'-AAGUCUCGUG CCUCGUAGA-3'). siCON or siAtg7 were synthesized by GenePharma (Shanghai, China). At $48 \mathrm{~h}$ after transfection, the cells were harvested for western blot analysis.

Protein extraction and western blot analysis. Cells were washed with cold phosphate-buffered saline (PBS) and with RIPA lysis solution [pH 7.5, 10\% glycerol, $30 \mathrm{mM}$ Tris- $\mathrm{HCl}$, $1 \%$ Triton $\mathrm{X}-100$ and $150 \mathrm{mM} \mathrm{NaCl}$ (Beyotime, Shanghai, China)] with protease inhibitors $(10 \mu \mathrm{g} / \mathrm{ml}$ pepstatin, $2 \mathrm{mM}$ EDTA, $100 \mu \mathrm{M}$ phenylmethylsulfonyl fluoride, and $10 \mu \mathrm{g} / \mathrm{ml}$ leupeptin), and then, they were incubated for $15 \mathrm{~min}$ at $4^{\circ} \mathrm{C}$ after the treatment. The cells were centrifuged at $12,000 \mathrm{x} \mathrm{g}$ for $10 \mathrm{~min}$ at $4^{\circ} \mathrm{C}$. The Bio-Rad protein assay kit (Bio-Rad Laboratories, Hercules, CA, USA) was used to determine the protein concentration of the supernatant fraction according to the manufacturer's instructions. An equivalent amount of overall protein was aliquoted into sample solution (Invitrogen) and boiled for $5 \mathrm{~min}$. Samples were loaded onto freshly cast $12 \%$ polyacrylamide gels. The proteins were transferred to a $0.2-\mu \mathrm{m}$ nitrocellulose transfer membrane (Millipore, Temecula, CA, USA) after electrophoresis. Then, membranes were blotted at $4^{\circ} \mathrm{C}$ with the suitable primary antibodies overnight. After incubation with suitable conjugated secondary antibodies, the samples were visualised via enhanced chemiluminescence detection (Pierce, Rockford, IL, USA). The ImageJ Gel Analysis tool was used for densitometric analysis.

Quantitative real-time PCR. Moloney murine leukaemia virus reverse transcriptase (Invitrogen) was used to generate cDNA, and total RNA was obtained using TRIzol reagent (Life Technologies, Gaithersburg, MD, USA). The following primers were utilised for the amplification: GAPDH (antisense) 5'-GCT CAG TGT AGC CCA GGA T-3' and (sense) 5'-ACT GCC ACC CAG AAG ACT-3'; Atg7 (antisense) 5'-CAC GGA AGC AAA CAA CTT CAA C-3' and Atg7 (sense) 5'-ATG ATC CCT GTA ACT TAG CCC A-3'. SYBR-Green-based quantitative real-time PCR was used for PCR amplification on an ABI Prism 7900 HT sequence detection system (Applied Biosystems). The following thermocycler conditions were used: $94^{\circ} \mathrm{C}$ for $3 \mathrm{~min}$ followed by 35 cycles of $58^{\circ} \mathrm{C}$ for $30 \mathrm{sec}, 72^{\circ} \mathrm{C}$ for $20 \mathrm{sec}$ and $94^{\circ} \mathrm{C}$ for $30 \mathrm{sec}$, as well as a final extension at $72^{\circ} \mathrm{C}$ for $5 \mathrm{~min}$. GAPDH was used as an internal control gene.

Immunofluorescence and confocal microscopy analysis. Cells seeded on coverslips were immobilised for $10 \mathrm{~min}$ with $4 \%(\mathrm{w} / \mathrm{v})$ paraformaldehyde (Sigma-Aldrich, St. Louis, MO, USA). Then, the cells were treated with $0.1 \%$ Triton X-100 (Sigma-Aldrich) for $10 \mathrm{~min}$ at room temperature and washed 

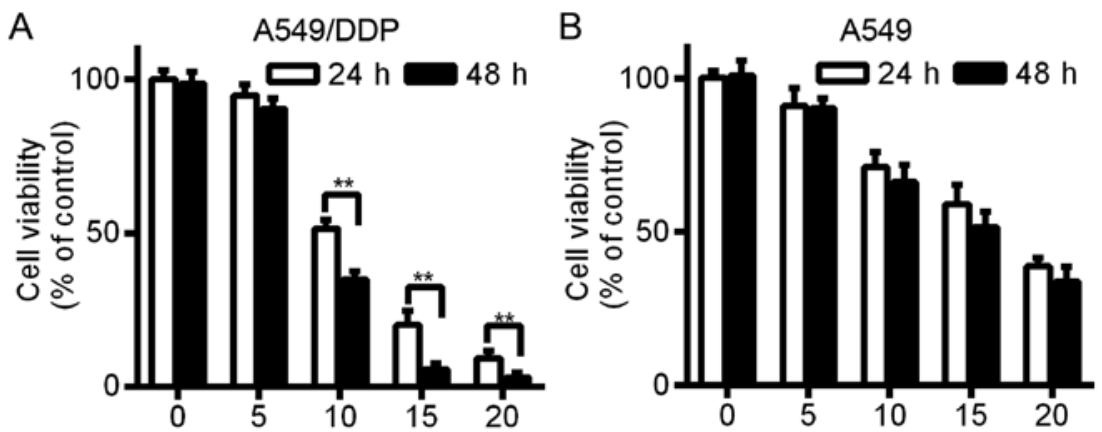

Figure 1. Viability of (A) A549/DDP and (B) A549 cells was inhibited by FTY720. Human lung carcinoma cells were treated with FTY720 at the indicated concentrations $(0,5,10,15$ and $20 \mu \mathrm{M})$ for 24 and $48 \mathrm{~h}$, and the survival rates were examined by CCK- 8 assay. FTY720 significantly decreased the viability of the two cell lines in a time- and dose-dependent manner. All data are shown as the mean \pm SD of 3 independent assays. In the untreated control cells, the cell viability was considered to be $100 \% ;{ }^{* *} \mathrm{P}<0.01$ vs. the indicated group; bars, SD.

with PBS and $10 \%(\mathrm{w} / \mathrm{v})$ goat serum and PBS containing $1 \%$ (w/v) bovine serum albumin (BSA) (both from Sigma-Aldrich) for $30 \mathrm{~min}$ at room temperature. The cells were subsequently incubated with primary antibodies at $4^{\circ} \mathrm{C}$ overnight, followed by incubation with the appropriate secondary antibodies for $1 \mathrm{~h}$ at room temperature. Coverslips were fixed via an anti-fade fixing solution including 4',6-diamidino-2-phenylindole (DAPI; Vector Laboratories, Burlingame, CA, USA) after the final washes with PBS. Images were obtained using a confocal microscope (Leica BMI-6000; Leica Microsystems, Cambridge, UK). Fields were selected based on DAPI staining for image quantification via Image J software, with a minimum 8 cells/image. Quantification of the number of LC3 dots for each cell was performed on at least 50 cells. The number of granules was quantified with the use of 'analyse granule' function.

Tumour xenografts in nude mice. Male BALB/c nude mice, 6 weeks old, were purchased from the Shanghai Experimental Animal Centre of the Chinese Academy of Sciences. All animal studies were performed in accordance with the guidelines of the Institutional Animal Care and Use Committee of Xi'an Jiaotong University. Before the experiments, all mice were acclimatised for 1 week and were housed with a $12 \mathrm{~h}$ lightdark cycle and a relative humidity of $55 \pm 5 \%$ at $25 \pm 2^{\circ} \mathrm{C}$. Then, every mouse underwent a subcutaneous flank injection with $1 \times 10^{7}$ A549/DDP cells in $0.1 \mathrm{ml}$ PBS. The mice were randomly divided into 3 treatment groups after 5 days: FTY720 $(5 \mathrm{mg} / \mathrm{kg}$ in saline, daily, gavage) plus DDP; vehicle PBS control; and DDP (1 mg/kg in saline, daily, gavage) alone. Treatments began on the day of random selection. Tumours were measured every 2 days using calipers and the tumour volume (V) was calculated with the following formula: $\mathrm{V}=$ width $^{2} \mathrm{x}$ length $/ 2$. Additionally, the weight of the neoplasms was calculated. The 3 groups received treatment by oral gavage every day starting on day 5. All animals were sacrificed on day 24. Mice in the control and treatment groups ( $n=5$ in each group) were sacrificed by cervical dislocation, and their neoplastic tissues were collected, snap-frozen and embedded in paraffin.

Immunohistochemistry. Based on a standard protocol, neoplasms were immobilized in formalin promptly and implanted in paraffin. On a cryotome, tissue sections were cut at a $5-\mu \mathrm{m}$ thickness. In activity antigen retrieval solution (10 mM citric buffer, $\mathrm{pH}$ 6.0), neoplasm sections were rehydrated, deparaffinised and heated in a microwave for $20 \mathrm{~min}$ for immunohistochemistry. Endogenous peroxidase was inactivated with $3 \%$ hydrogen peroxide solution. At room temperature, slides were blocked in $10 \%$ normal goat serum (Sigma-Aldrich) in PBS for $1 \mathrm{~h}$. Subsequently, the slides were incubated with primary antibody against Atg7 (1:50 dilution, rabbit polyclonal antibody) or Ki67 (1:50 dilution, rabbit polyclonal antibody) (both from Abcam, Cambridge, MA, USA) overnight in a humidity chamber at $4^{\circ} \mathrm{C}$. A secondary antibody (1:50 dilution, goat-anti-rabbit $\mathrm{IgG}$; Abcam) was incubated with the slides for $1 \mathrm{~h}$ at room temperature after washing with PBS. Then, cells were stained with 3,3'-diaminobenzidine (DAB) and cultured with strepavidin-biotin complex for $30 \mathrm{~min}$ at $37^{\circ} \mathrm{C}$. Sections were rinsed with Tris-buffered saline (TBS) 3 times for $10 \mathrm{sec}$ after the culture. Photomicrographs were captured on a Leica microscope assembled with a CCD camera before counterstaining with a quick staining kit (Zhongshan Jinqiao Biotechnology, Beijing, China).

Statistical analysis. All data are expressed as the mean \pm standard deviation (SD). Two-tailed homoscedastic Student's t-test was used to determine the significance of differences between two groups. All data were assessed with SPSS statistical software (for Windows, version 18.0; SPSS, Inc., Chicago, IL, USA).

\section{Results}

FTY720 inhibits the growth of A549/DDP and A549 cells. A549 and A549/DDP cells were exposed to various concentrations of FTY720 $(0,5,10,15$ and $20 \mu \mathrm{M})$ for 24 and $48 \mathrm{~h}$. Cell survival was detected with CCK-8 assays. The findings indicated that A549 and A549/DDP cell viability was inhibited by FTY720 in a time- and dose-dependent manner (Fig. 1; P<0.01). After FTY720 treatment, the $\mathrm{IC}_{50}$ value of the A549 and A549/DDP cells was $17.21 \pm 4.23$ and $10.98 \pm 1.45 \mu \mathrm{M}$, respectively.

FTY720 induces A549/DDP cell apoptosis in a time- and dosedependent manner. The cisplatin-resistant A549/DDP cells were treated with $0,5,10$ and $20 \mu \mathrm{M}$ FTY720 for $24 \mathrm{~h}$, and $10 \mu \mathrm{M}$ FTY720 for $0,12,24$ and $48 \mathrm{~h}$. After treatment with FTY720 at 5, 10 and $20 \mu \mathrm{M}$, respectively, for $24 \mathrm{~h}$, the cell apoptosis rate was $4.17 \pm 1.02,54.46 \pm 8.23$ and $70.27 \pm 9.45 \%$, which was significantly different from the group that was not treated with FTY720 (1.02 $\pm 0.54 \%)$ (Fig. 2A; P<0.01). There was an 

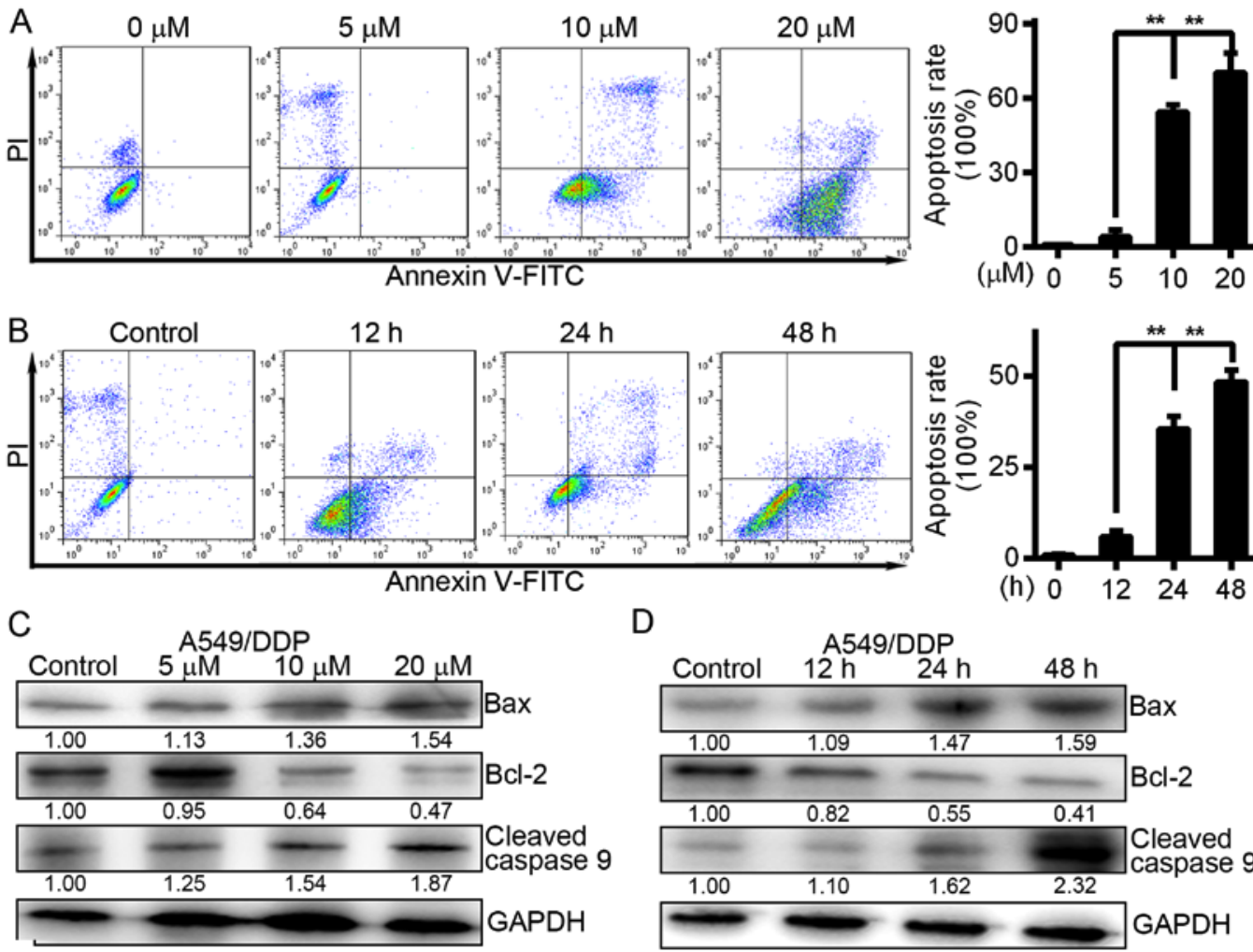

Figure 2. FTY720 induces the apoptosis of A549/DDP cells in a time- and dose-dependent manner. (A) A549/DDP cells were treated with FTY720 at the indicated concentrations $(0,5,10$ and $2.0 \mu \mathrm{M})$ for $24 \mathrm{~h}$. The apoptotic rates of cells were quantified. (B) A549/DDP cells were treated with $10 \mu \mathrm{M}$ FTY720 for $0,12,24$ and $48 \mathrm{~h}$. Cell apoptosis was analysed at different time points by flow cytometry. The rates of cell apoptosis were quantified. (C) A549/DDP cells were treated with FTY720 at the indicated concentrations $(0,5,10$ and $20 \mu \mathrm{M})$ for $24 \mathrm{~h}$. (D) A549/DDP cells were treated with FTY720 for 0,12, 24 and $48 \mathrm{~h}$. The levels of cleaved caspase-9, Bcl-2 and Bax were assessed by western blotting. The data are presented as the mean of independent assays; ${ }^{* *} \mathrm{P}<0.01 ;$ bars, SD.
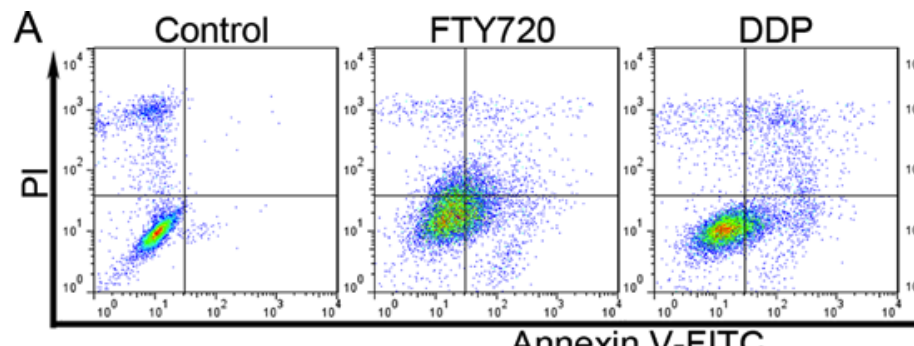

FTY720+DDP
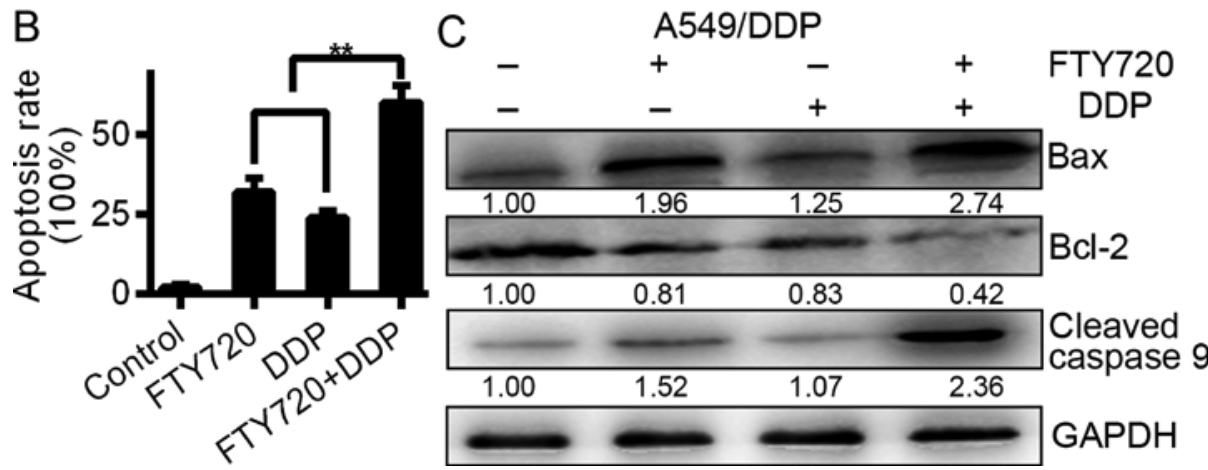

Figure 3. FTY720 combined with DDP enhances the apoptosis rate of A549/DDP cells. (A and B) A549/DDP cells were treated with FTY720 (10 $\mu$ M) combined with DDP $(10 \mu \mathrm{g} / \mathrm{ml})$ or DDP or FTY720 alone for $24 \mathrm{~h}$. (C) A549/DDP cells were treated with DDP or FTY720 alone or FTY720 (10 $\mu \mathrm{M})$ in combination with DDP $(10 \mu \mathrm{g} / \mathrm{ml})$ for $24 \mathrm{~h}$. The levels of cleaved caspase-9, Bax and Bcl-2 were assessed by western blot experiments. Data are presented as the mean \pm SD from 3 independent assays; ${ }^{* *} \mathrm{P}<0.01$; bars, $\mathrm{SD}$.

increase in A549/DDP cell apoptosis after 12-48 $\mathrm{h}$ of treatment with FTY720. The findings showed that A549/DDP cell apoptosis was induced by FTY720 in a time- and dose-dependent manner. We assessed protein expression in A549/DDP cells and found that the $\mathrm{Bcl}-2$ protein level was downregulated while the levels of cleaved caspase- 9 and Bax were upregulated following 

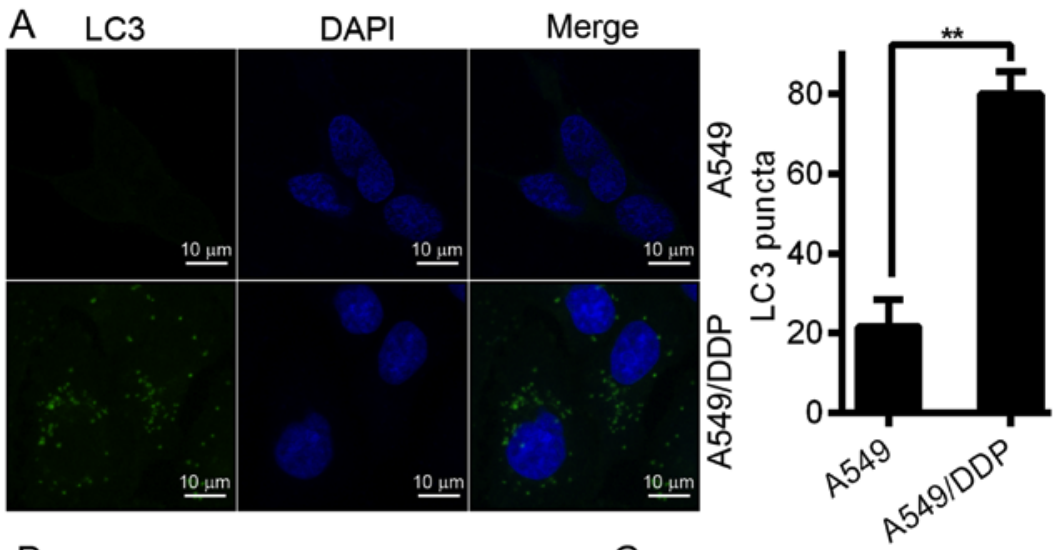

B

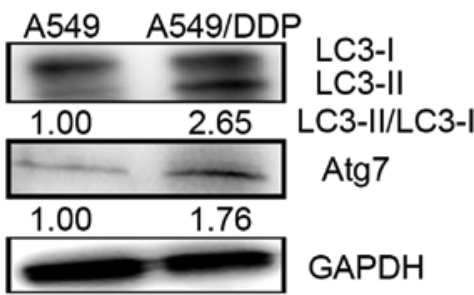

C

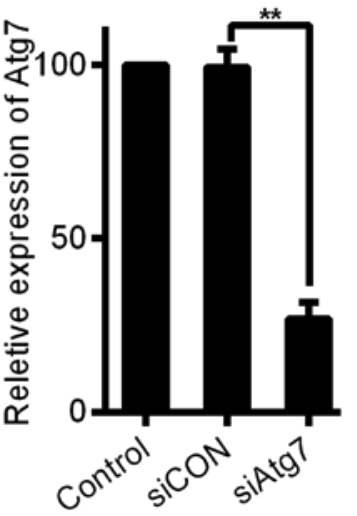

Figure 4. Difference in autophagy levels between A549/DDP and A549 cells. (A) LC3 antibody-stained A549/DDP or A549 cells. The percentage of LC3positive cells showing representative LC3 puncta ( $>5$ puncta/cell) is shown (right panel), and typical images of LC3 staining are presented (left panels). Data are the mean \pm SD of 3 independent assays ( $\geq 100$ cells were counted in every assay). (B) Western blot analysis of the expression levels of Atg7, LC3-II and LC3-I. (C and D) Cells were transfected with siCON or Atg7 siRNA, and the Atg7 expression was assessed by western blotting and real-time PCR. GAPDH was used as a loading control; ${ }^{* *} \mathrm{P}<0.01$; bars, SD..

FTY720 treatment (Fig. 2C and D), further confirming that A549/DDP cell apoptosis was induced by FTY720.

FTY720 combined with DDP enhances the cell apoptosis of A549/DDP cells. The cisplatin-resistant A549/DDP cells were treated with a combination of FTY720 $(10 \mu \mathrm{M})$ and DDP $(10 \mu \mathrm{g} / \mathrm{ml})$. The apoptosis rates of the A549/DDP cells were $31.82 \pm 7.56,23.90 \pm 4.32$ and $60.07 \pm 6.56 \%$ for the DDP monotreatment, control and combination treatment groups, respectively, after 1 day. However, the combination treatment group showed increased apoptosis compared to the monotreatment and control groups (Fig. 3A and 3B; $\mathrm{P}<0.01$ ). Furthermore, expression levels of Bax, cleaved caspase- 9 and Bcl-2 were determined using western blot analysis after the combination treatment. The findings indicated that the combination of the two agents resulted in a significantly greater decrease in Bcl-2 and increase in Bax and cleaved caspase- 9 than that of either drug alone (Fig. 3C; $\mathrm{P}<0.01$ ). Collectively, those findings indicated that DDP in combination with FTY720 showed increased efficacy through apoptosis induction in A549/DDP cells.

DDP-resistant cells exhibit increased levels of autophagy. We considered that autophagy formation may be a mechanism underlying DDP resistance. First, we determined the potential effect of DDP on autophagy in lung carcinoma cells to verify this assumption. Notably, we observed accretion of LC puncta in the A549/DDP cells via immunofluorescence (Fig. 4A).
Based on the number of cells with fluorescent puncta, a substantial increase was observed in the resistant A549/DDP cells. However, in A549 cells, the marker LC3 showed a dispersed distribution via fluorescence. Compared with the sensitive A549 cells, A549/DDP, which is a DDP-resistant cell line, showed a substantial increase in baseline levels of autophagy, as determined by LC3 expression, confirming the difference in autophagy levels between DDP-sensitive and -resistant cells (Fig. 4B). Western blot analyses were carried out to assess the effect on autophagy and Atg7 in chemoresistance. Increased Atg7 induction was displayed in the A549/DDP cells with high levels of autophagy as shown in Fig. 4B (middle panel). Compared with the DDP-sensitive cells, DDP-resistant lung carcinoma cells showed increased levels of autophagy.

Silencing of Atg7 sensitises chemoresistant cells to drug treatment. We used siRNAs to knockdown the Atg7 expression to further verify that Atg7 promotes drug resistance of lung carcinoma cells. One siRNA was used. Western blot analysis and real-time PCR showed that Atg7 expression was inhibited by the siRNA at both the mRNA and protein level (Fig. 4C and D). We knocked down the expression of Atg7 and subsequently treated the A549/DDP cells with 3-MA or FTY720. After the treatment, cell apoptosis was assessed at 24 h. Compared with siCON, Atg7 knockdown resulted in an increase in the apoptosis rate of the A549/DDP cells. Compared with cells treated with siAtg7 alone for 1 day after treatment, 
A

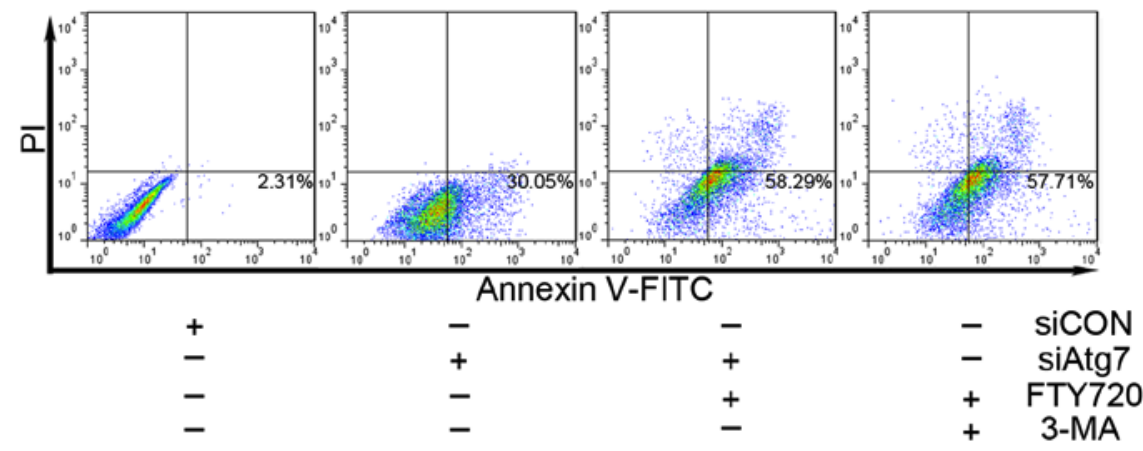

B
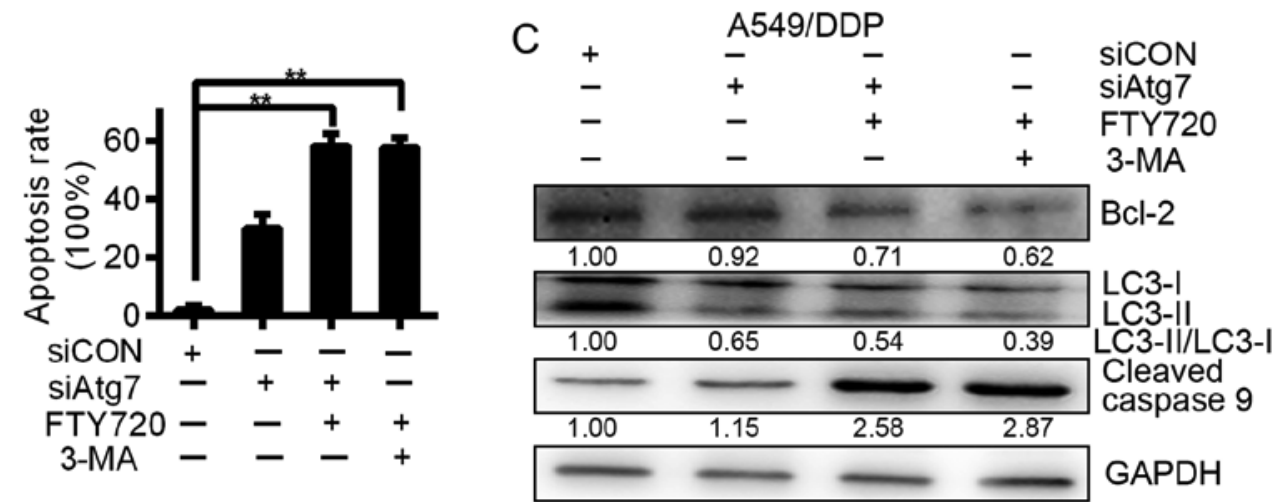

Figure 5. Silencing of Atg7 sensitises chemoresistant cells to agent treatment. (A and B) After diverse treatments, the rate of apoptosis was examined by FACS analysis for $24 \mathrm{~h}$. (C) A549/DDP cells were treated with FTY720, 3-MA or siAtg7 for $24 \mathrm{~h}$, and subsequently, the cell lysates were collected. The protein expression of LC3-I/II, cleaved caspase- 9 and Bcl-2 was assessed by western blotting. The protein expression was standardised to that of GAPDH; ** P $<0.01$; bars, SD. FACS, fluorescence-activated cell-sorting.

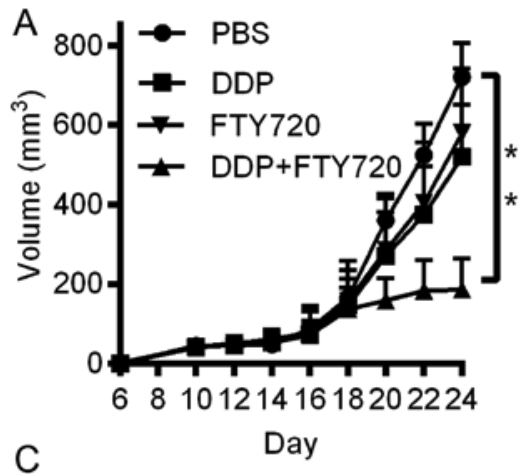

B
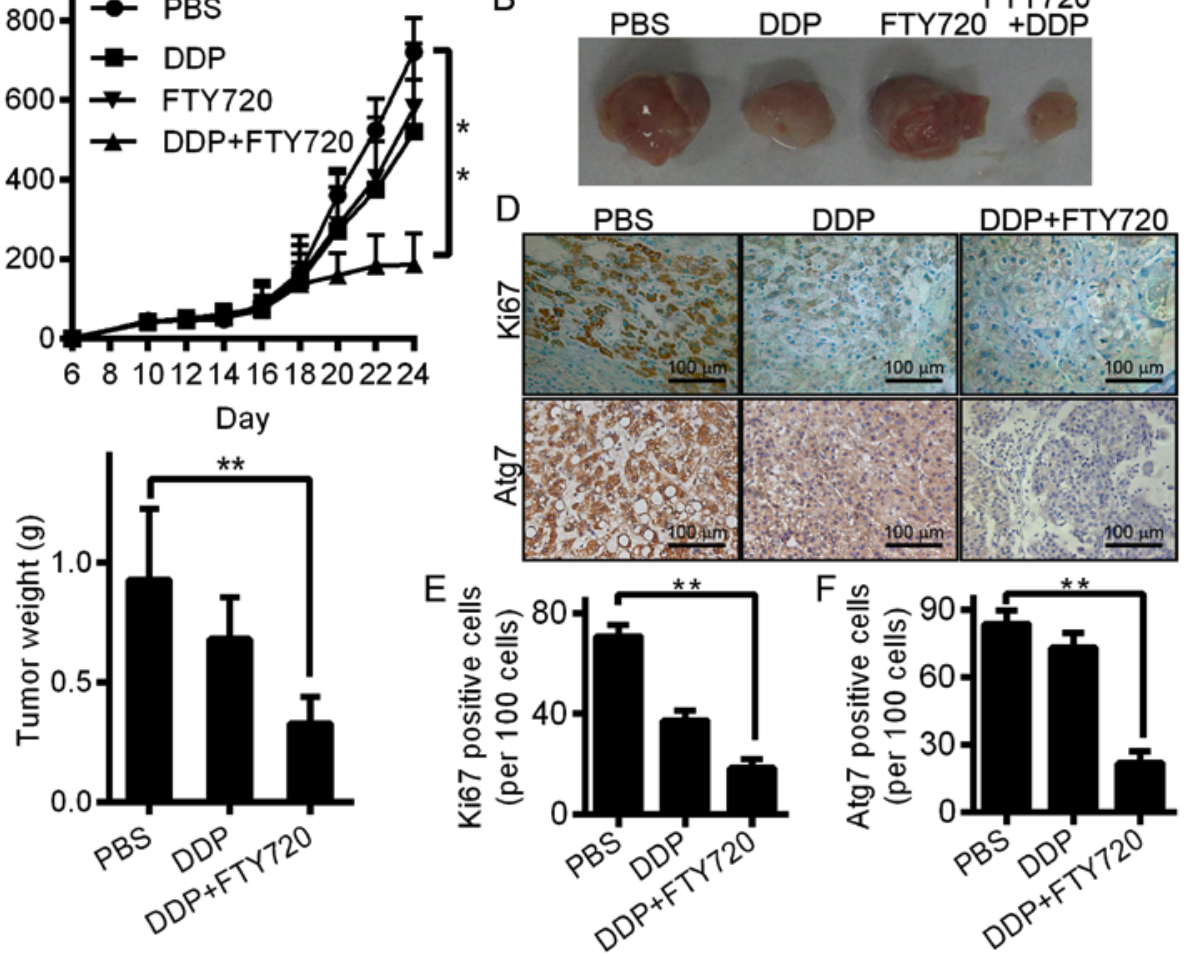

Figure 6. In mice bearing lung carcinoma, FTY720 improves the anticancer activity of DDP. (A) A xenograft model was constructed using 6-week-old $\mathrm{BALB} / \mathrm{c}$ nude mice. The tumour volume curves of the mice treated with DDP $(1 \mathrm{mg} / \mathrm{kg}$, daily, gavage) alone, FTY720 (5 mg/kg, daily, gavage) and DDP (n=5/ group), or PBS control are shown; ${ }^{* *} \mathrm{P}<0.01$ vs. control. (B) Photomicrographs of xenograft tumours from nude mice. In every group, typical images of a mouse are shown. (C) The weights of tumour for the 3 groups of BALB/c nude mice. Data are shown as the mean \pm SD $(n=5) ;{ }^{* *} \mathrm{P}<0.01$ vs. PBS control groups. (D) Tumours were immunostained for the indicated molecules in all groups. Cell expression of Ki-67 was quantified. Atg7 was stained and quantified as a marker of autophagy. Images are typical of 3 independent assays. (E) After immunostaining, the percenage of Ki67-positive cells is presented. (F) After immunostaining, the percentage of Ki67-positive cells is presented. In tumour tissues, positive cells were calculated and are shown as the mean \pm SD (3 sections/tumour and 5 fields at random/section); ${ }^{* *} \mathrm{P}<0.01$ 
apoptosis was further increased when we knocked down Atg7 expression and treated the cells with 3-MA or FTY720 simultaneously (Fig. 5A and B). Our data further confirm that Atg7 promotes drug resistance of lung carcinoma cells.

FTY720 increases the antitumour activity of DDP in lung cancer-bearing mice. We established a xenograft lung carcinoma model in nude mice to further investigate the antitumour effects of DDP combined with FTY720. At the end of the experiment, all mice survived and did not show any change in body temperature and general toxicity. Mice were allocated at random to the post tumour cell inoculation, PBS and DDP $(1 \mathrm{mg} /$ $\mathrm{kg}$, daily, gavage) alone groups 6 days post tumour cell injection (Fig. 6A). An important decrease in tumour size resulted from the treatment of FTY720 plus DDP compared with the control group (Fig. 6A and B). Additionally, in the group treated with FTY720 plus DDP, the tumour weight of the mice was less than that of the control group (Fig. 6C), which indicated that FTY720 inhibited the development of lung carcinoma. After the mice were sacrificed, their tumours were collected, and Ki67 and Atg7 expression was assessed. Immunohistochemistry showed that variations in Atg7 and Ki67 reflected the cell autophagy and proliferation of tumours (Fig. 6D-F). Compared with PBS control mice, the mice with lung carcinoma that were treated with FTY720 and DDP showed decreased expression of Atg7 and Ki67. Moreover, the efficacy of the antitumour effect of DDP combined with FTY720 was further improved. Therefore, our results suggest that the anticancer activity of DDP can be increased by FTY720.

\section{Discussion}

A major obstacle to the successful treatment of patients with carcinoma is drug efficacy. In the treatment of lung carcinoma, one of the major obstacles is still resistance to chemotherapy based on DDP (17). Therefore, it is essential to identify new drugs to overcome DDP resistance. Similarly, one of the major methods for improving treatment results in ovarian carcinoma is DDP treatment combined with numerous modalities or drugs to overcome drug resistance $(18,19)$. We reported in the present study that the viability of DDP-resistant A549/DDP cells could be effectively inhibited by FTY720 in a time- and dose-dependent manner (Fig. 1A). Notably, compared with the A549/DDP cells, A549 cells displayed a higher survival rate after FTY720 treatment at 10, 15 and $20 \mu \mathrm{M}$ for $48 \mathrm{~h}$ (Fig. 1B). This unexpected phenomenon is worth further exploration, although there was no major effect on our conclusions. We hypothesised that compared with A549 cells, the A549/DDP cells showed enhanced sensitivity to FTY720.

A549/DDP cells, which have DDP resistance, have diverse protein and gene expression levels. The A549/DDP phenomenon may offer another viewpoint on DDP resistance in lung carcinoma. Further research showed that by decreasing cell apoptosis (Fig. 2) of A549/DDP cells, FTY720 may prevent cell growth. One of the limitations of the present study was that it could not account for the cell apoptosis phenomenon by FTY720 on A549/DDP cells.

We assessed the influence of FTY720 in combination with DDP on A549/DDP cells to clarify whether FTY720 reversed DDP resistance in lung carcinoma. Based on our findings, the apoptosis of A549/DDP cells was substantially increased by FTY720 in combination with DDP (Fig. 3). Similarly, it was also reported in other studies that FTY720 improved the sensitivity of NSCLC cells to paclitaxel with a new SET inhibitor, EMQA (20). Moreover, compared with the single drug treatments in A549/DDP cells, the rate of apoptosis was higher when FTY720 was used in combination with DDP (Fig. 3). As a result, we hypothesised that FTY720 improved the cytotoxic effect of DDP against lung carcinoma, which is DDP-resistant.

Since autophagy has been proposed to be a significant method of protection against anticancer treatments, we studied the effect of autophagy to identify possible methods by which FTY720 enhances DDP cytotoxicity $(21,22)$. In fact, previous studies have demonstrated that FTY720 can induce the process of autophagy and improve autophagic flux in carcinoma cells $(23,24)$. Genes related to autophagy are involved in many types of autophagy whose functions depend on cellular context. Thus, we hypothesised that the combination of DDP with FTY720 may overcome the resistance due to the protective function of autophagy in response to DDP treatment, which is difficult to manage. Our results supported this hypothesis. To influence and allow the conjugation of ATG12 to ATG5 and then improve the formation of lipid phosphatidylethanolmine, the E2-like enzyme ATG10 cooperates with the E1-like enzyme ATG7 (25). In the mouse liver, Atg7 loss ameliorates the development of HCC (26). Conversely, the growth of breast tumours induced by oncogenic KRAS was prevented by the inhibition of ATG7 (27), and for breast carcinoma, high levels of ATG7 predict a poor prognosis (28). Additionally, in most samples of HCC, upregulation of ATG7 was shown, and during metabolic stress, tumour cell survival was promoted (29). As determined by LC3 staining and the LC3-II expression levels, under drugstimulated and basal conditions, the level of autophagy was detected in the DDP-resistant A549/DDP cells, which was higher than that in the sensitive A549 cells (Fig. 4). Notably, in A549/DDP carcinoma cells, prevention of autophagy enhanced the cytotoxicity of 3-MA, a small-molecule inhibitor. The killing effect of FTY720 was strongly promoted by the obstruction of autophagy formation due to the siRNA-mediated knockdown of Atg7. Moreover, 3-MA inhibition of autophagy allowed the combination of FTY720 and DDP to kill lung carcinoma cells (Fig. 5). The observed results demonstrate that following treatment of FTY720 in combination with DDP, autophagy was at least partly responsible for the killing effect of FTY720, and these findings also show the influence of FTY720 in the induction of autophagy. According to current research, in mantle cell lymphoma cell therapy, an important collaborative influence resulted from the combination of FTY720 with milatuzumab, which is an anti-CD74 mAb. The collaborative effect of FTY720 was attributed to blockage of destruction of the autophagiclysosomal regression of CD74 and autophagic flux (21). On the basis of the present study, autophagic flux, which leads to a contradictory increase in autophagosomes in apoptotic breast carcinoma cells, was prevented by the combination of FTY720 and $\gamma$-irradiation (30). In lymphoma cells, the influence of FTY720 on autophagy corresponded to our research. However, according to other studies, FTY720 improves autophagic flux and induces the formation of autophagy in ovarian carcinoma cells and those of other organs (24). These differences may indicate that the influence of FTY720 on autophagy is cell 
type-dependent. Notably, previous studies have demonstrated that autophagy functions as either a pro-death or a pro-survival mechanism depending on the context (31-35). To further elucidate the observed results, more studies should be performed. Similarly, the effects of FTY720 on autophagy should be considered in assessment of the anticancer effectiveness of FTY720, particularly in combination with other chemotherapeutic drugs.

In conclusion, we demonstrated that FTY720 exhibited excellent anti-growth effects by inducing the apoptosis of NSCLC cells. In A549/DDP human lung carcinoma cells, which are DDP-resistant, downregulation of Atg7 may be a mechanism underlying the effects of FTY720. Via downregulation of the expression of Atg7, FTY720 combined with DDP had enhanced antitumour effects on DDP-resistant lung carcinoma cells. Collectively, we demonstrated inhibition of tumour development in vivo and in vitro and identified a new mechanism by which FTY720 enhances apoptosis induced by DDP in human NSCLC.

\section{Acknowledgements}

The present study was supported by the Shaanxi Science and Technology Research Funds (grant no. S2015YFSF0297).

\section{References}

1. Siegel RL, Miller KD and Jemal A: Cancer statistics, 2015. CA Cancer J Clin 65: 5-29, 2015

2. Molina JR, Yang P, Cassivi SD, Schild SE and Adjei AA: Non-small cell lung cancer: Epidemiology, risk factors, treatment, and survivorship. Mayo Clin Proc 83: 584-594, 2008.

3. Reed JC: Mechanisms of apoptosis avoidance in cancer. Curr Opin Oncol 11: 68-75, 1999.

4. Judson I and Kelland LR: New developments and approaches in the platinum arena. Drugs 59 (Suppl 4): S29-S38, 2000.

5. Rosell R, Lord RV, Taron $M$ and Reguart N: DNA repair and cisplatin resistance in non-small-cell lung cancer. Lung Cancer 38: 217-227, 2002.

6. Duan S, Tsai Y, Keng P, Chen Y, Lee SO and Chen Y: IL-6 signaling contributes to cisplatin resistance in non-small cell lung cancer via the up-regulation of anti-apoptotic and DNA repair associated molecules. Oncotarget 6: 27651-27660, 2015.

7. Zhang N, Qi Y, Wadham C, Wang L, Warren A, Di W and Xia P: FTY720 induces necrotic cell death and autophagy in ovarian cancer cells: A protective role of autophagy. Autophagy 6: 1157-1167, 2010.

8. Hung JH, Lu YS, Wang YC, Ma YH, Wang DS, Kulp SK, Muthusamy N, Byrd JC, Cheng AL and Chen CS: FTY720 induces apoptosis in hepatocellular carcinoma cells through activation of protein kinase C delta signaling. Cancer Res 68: 1204-1212, 2008.

9. Yasui H, Hideshima T, Raje N, Roccaro AM, Shiraishi N, Kumar S, Hamasaki M, Ishitsuka K, Tai YT, Podar K, et al: FTY720 induces apoptosis in multiple myeloma cells and overcomes drug resistance. Cancer Res 65: 7478-7484, 2005.

10. Neviani P,Santhanam R, Oaks JJ, Eiring AM, Notari M,Blaser BW, Liu S, Trotta R, Muthusamy N, Gambacorti-Passerini C, et al: FTY720, a new alternative for treating blast crisis chronic myelogenous leukemia and Philadelphia chromosome-positive acute lymphocytic leukemia. J Clin Invest 117: 2408-2421, 2007.

11. Estrada-Bernal A, Palanichamy K, Ray Chaudhury A and Van Brocklyn JR: Induction of brain tumor stem cell apoptosis by FTY720: A potential therapeutic agent for glioblastoma. Neuro-oncol 14: 405-415, 2012.

12. Azuma H, Takahara S, Ichimaru N, Wang JD, Itoh Y, Otsuki Y, Morimoto J, Fukui R, Hoshiga M, Ishihara T, et al: Marked prevention of tumor growth and metastasis by a novel immunosuppressive agent, FTY720, in mouse breast cancer models. Cancer Res 62: 1410-1419, 2002.

13. Amaravadi RK, Lippincott-Schwartz J, Yin XM, Weiss WA, Takebe N, Timmer W, DiPaola RS, Lotze MT and White E: Principles and current strategies for targeting autophagy for cancer treatment. Clin Cancer Res 17: 654-666, 2011.

14. Klionsky DJ and Emr SD: Autophagy as a regulated pathway of cellular degradation. Science 290: 1717-1721, 2000.
15. Degenhardt K, Mathew R, Beaudoin B, Bray K, Anderson D, Chen G, Mukherjee C, Shi Y, Gélinas C, Fan Y, et al: Autophagy promotes tumor cell survival and restricts necrosis, inflammation, and tumorigenesis. Cancer Cell 10: 51-64, 2006.

16. Jin $\mathrm{S}$ and White E: Tumor suppression by autophagy through the management of metabolic stress. Autophagy 4: 563-566, 2008.

17. Wang Y, Wen L, Zhao SH, Ai ZH, Guo JZ and Liu WC: FoxM1 expression is significantly associated with cisplatin-based chemotherapy resistance and poor prognosis in advanced non-small cell lung cancer patients. Lung Cancer 79: 173-179, 2013.

18. Dilruba S and Kalayda GV: Platinum-based drugs: Past, present and future. Cancer Chemother Pharmacol 77: 1103-1124, 2016.

19. Fennell DA, Summers Y, Cadranel J, Benepal T, Christoph DC, Lal R, Das M, Maxwell F, Visseren-Grul C and Ferry D: Cisplatin in the modern era: The backbone of first-line chemotherapy for non-small cell lung cancer. Cancer Treat Rev 44: 42-50, 2016.

20. Hung MH, Wang CY, Chen YL, Chu PY, Hsiao YJ, Tai WT, Chao TT, Yu HC, Shiau CW and Chen KF: SET antagonist enhances the chemosensitivity of non-small cell lung cancer cells by reactivating protein phosphatase 2A. Oncotarget 7: 638-655, 2016.

21. Alinari L, Baiocchi RA and Praetorius-Ibba M: FTY720-induced blockage of autophagy enhances anticancer efficacy of milatuzumab in mantle cell lymphoma: Is FTY720 the next autophagy-blocking agent in lymphoma treatment? Autophagy 8: 416-417, 2012.

22. White C, Alshaker H, Cooper C, Winkler M and Pchejetski D: The emerging role of FTY720 (Fingolimod) in cancer treatment. Oncotarget 7: 23106-23127, 2016.

23. Leu WJ, Swain ShP, Chan SH, Hsu JL, Liu SP, Chan ML, Yu CC, Hsu LC, Chou YL, Chang WL, et al: Non-immunosuppressive triazole-based small molecule induces anticancer activity against human hormone-refractory prostate cancers: The role in inhibition of $\mathrm{PI} 3 \mathrm{~K} / \mathrm{AKT} / \mathrm{mTOR}$ and c-Myc signaling pathways. Oncotarget 7: 76995-77009, 2016.

24. Zhang N, Dai L, Qi Y, Di W and Xia P: Combination of FTY720 with cisplatin exhibits antagonistic effects in ovarian cancer cells: Role of autophagy. Int J Oncol 42: 2053-2059, 2013.

25. Xie Z and Klionsky DJ: Autophagosome formation: Core machinery and adaptations. Nat Cell Biol 9: 1102-1109, 2007.

26. Takamura A, Komatsu M, Hara T, Sakamoto A, Kishi C, Waguri S, Eishi Y, Hino O, Tanaka K and Mizushima N: Autophagy-deficient mice develop multiple liver tumors. Genes Dev 25: 795-800, 2011.

27. Guo JY, Chen HY, Mathew R, Fan J, Strohecker AM, KarsliUzunbas G, Kamphorst JJ, Chen G, Lemons JM, Karantza V, et al: Activated Ras requires autophagy to maintain oxidative metabolism and tumorigenesis. Genes Dev 25: 460-470, 2011.

28. Desai S, Liu Z, Yao J, Patel N, Chen J, Wu Y, Ahn EE, Fodstad O and Tan M: Heat shock factor 1 (HSF1) controls chemoresistance and autophagy through transcriptional regulation of autophagyrelated protein 7 (ATG7). J Biol Chem 288: 9165-9176, 2013.

29. Chang Y, Yan W, He X, Zhang L, Li C, Huang H, Nace G, Geller DA, Lin J and Tsung A: miR-375 inhibits autophagy and reduces viability of hepatocellular carcinoma cells under hypoxic conditions. Gastroenterology 143: 177-187.e8, 2012.

30. Pereira FV, Arruda DC, Figueiredo CR, Massaoka MH, Matsuo AL, Bueno V and Rodrigues EG: FTY720 induces apoptosis in B16F10-NEX2 murine melanoma cells, limits metastatic development in vivo, and modulates the immune system. Clinics 68: 1018-1027, 2013.

31. Shi Y, Han JJ, Tennakoon JB, Mehta FF, Merchant FA, Burns AR, Howe MK, McDonnell DP and Frigo DE: Androgens promote prostate cancer cell growth through induction of autophagy. Mol Endocrinol 27: 280-295, 2013.

32. Deng L, Lei Y, Liu R, Li J, Yuan K, Li Y, Chen Y, Liu Y, Lu Y, Edwards CK III, et al: Pyrvinium targets autophagy addiction to promote cancer cell death. Cell Death Dis 4: e614, 2013.

33. Patel S, Hurez V, Nawrocki ST, Goros M, Michalek J, Sarantopoulos J, Curiel T and Mahalingam D: Vorinostat and hydroxychloroquine improve immunity and inhibit autophagy in metastatic colorectal cancer. Oncotarget 7: 59087-59097, 2016.

34. Gong J, Muñoz AR, Chan D, Ghosh R and Kumar AP: STAT3 down regulates LC3 to inhibit autophagy and pancreatic cancer cell growth. Oncotarget 5: 2529-2541, 2014.

35. Dando I, Donadelli M, Costanzo C, Dalla Pozza E, D'Alessandro A, Zolla L and Palmieri M: Cannabinoids inhibit energetic metabolism and induce AMPK-dependent autophagy in pancreatic cancer cells. Cell Death Dis 4: e664, 2013. 